\title{
Best evidence in critical care medicine
}

\section{Treatment for hyperglycemia in the intensive care unit: a "bittersweet" message}

\section{Article appraised}

Van den Berghe $G$, Wilmer A, Hermans $G$, et al. Intensive insulin therapy in the medical ICU. N Engl J Med 2006; 354; 5: 449-61.

\section{Structured abstract}

Background: Hyperglycemia and insulin resistance are common in severe illness and are associated with a worse outcome. ${ }^{1}$ In 2001, a randomized, single-centre, prospective unblinded trial of surgical patients compared intensive-glycemic control (serum glucose 4.4-6.1 mmol. $\mathrm{L}^{-1}$ ) with more liberal glucose management (serum glucose $\left.10-12 \mathrm{mmol} \cdot \mathrm{L}^{-1}\right) .^{2}$ Significant decreases in mortality and morbidity were observed in the intensively treated group. In addition, a pronounced mortality benefit was demonstrated for patients who required intensive care unit (ICU) therapy for three or more days. It was unresolved as to whether or not these benefits may apply to medical ICU patients. This study was undertaken to address these issues.

Question: Does intensive insulin treatment decrease mortality amongst medical ICU patients?

Design: Single centre, prospective, non-blinded, randomized controlled trial comparing conventional insulin therapy to intensive insulin therapy in medical ICU patients expected to stay in ICU for three or more days. The study was conducted between 2002 and 2005.

Patients: One thousand two-hundred patients admitted to a medical ICU in Leuven, Belgium and, for whom the anticipated length was > three days, were enrolled. Postoperative surgical patients and those with do-not-resuscitate orders were excluded. Of patients enrolled, 767 remained in ICU for at least three days. The investigation was powered to detect a difference of $7 \%$ in outcome (based upon the investigators' previous results in surgical ICU patients). The two groups were similar in all respects, apart from their baseline Therapeutic Intervention Scoring System-28 (TISS-28) scores, which were higher in the treatment group.
Intervention: Patients in the intensive-treatment group received an iv insulin infusion if admission serum glucose concentration was $>6.1 \mathrm{mmol} \cdot \mathrm{L}^{-1}$. The infusion was titrated to maintain a target glucose concentration of 4.4 to $6.1 \mathrm{mmol} \cdot \mathrm{L}^{-1}$. Patients in the conventional-treatment group received $i v$ insulin infusions when admission serum glucose concentration exceeded > $12 \mathrm{mmol} \cdot \mathrm{L}^{-1}$. This infusion was titrated to maintain a blood glucose concentration of 10-11 $\mathrm{mmol} \cdot \mathrm{L}^{-1}$.

Primary outcome: All-cause, in-hospital, mortality.

Secondary outcomes: Mortality in ICU; 90-day mortality; days to weaning from mechanical ventilation; initiation of dialysis; and others.

\section{Results}

Mortality: Amongst the 1,200 patients included in the intention-to-treat analysis there was no significant difference in mortality $(37.3 \%$ in the intensive-treatment group vs $40.0 \%$ in the conventional-treatment group, $P=0.33$ ). However, amongst the 767 patients who remained in ICU for three or more days, all-cause in-hospital mortality was lower in the intensive-treatment group $(43.0 \%$ vs $52.5 \%, P=0.009)$. While more deaths $(n=56)$ occurred in the intensive treatment group among patients who stayed in ICU for less than three days, this number was not significantly different from the number of deaths in the conventional treatment group $(n=42)$.

Morbidity: Intensive insulin therapy resulted in significant morbidity benefits; as determined by accelerated weaning from mechanical ventilation; accelerated discharge from both the ICU and hospital; and a decrease in newly-acquired renal dysfunction. This benefit was seen in patients who stayed in ICU less than three days, and also in patients who stayed at least three days. In the intention-to-treat analysis, 111 of the patients who received intensive-insulin therapy experienced hypoglycemic episodes (serum glucose $<2.2 \mathrm{mmol} \cdot \mathrm{L}^{-1}$ ) vs 19 in the conventional treatment group. 
Conclusion: For patients staying in the ICU longer than three days, maintaining blood glucose concentrations between 4.4-6.1 mmol. $\mathrm{L}^{-1}$ during ICU admission is associated with reduced in-hospital mortality, but more frequent hypoglycemic events. Intensive insulin therapy significantly reduced morbidity, but not mortality amongst all medical ICU patients.

\section{Commentary}

It had been appreciated for over a century that severe illness is associated with hyperglycemia. However, for most of that time "stress-hyperglycemia" has been either largely ignored, or assumed adaptive. It is now well established that both the degree and duration of hyperglycemia are independent risk factors for adverse outcome: whether for patients following severe braininjury, severe pediatric-burns, critical-illness polyneuropathy, trauma, myocardial infarction, stroke, as well as for a heterogeneous group of ICU patients whether previously diagnosed diabetic or not. ${ }^{1-5}$ What remains to be determined is whether or not therapeutic euglycemia is associated with improved outcome. The study of Van den Berghe et al. provides important new information to help resolve this question.

The previous work of this group involving surgical and trauma ICU patients ${ }^{2}$ has influenced clinical practice, and heightened our awareness regarding seemingly mundane interventions such as glycemic control. Notably, for many, 'tight' glycemic control has since become an indicator of quality of care in the ICU. It also helped to emphasize a new paradigm where resuscitation advances well beyond the concept of simple insertion of "lines and tubes". This study will influence our approach to the care of medical ICU patients. However, in context of the current literature, intensive-insulin therapy is not beneficial for all; nor is it innocuous.

Prior to the study of Van den Berghe et al., many clinicians made the specious assumption that therapeutic interventions for surgical ICU patients might freely translate to medical ICU patients. An important lesson from this study is to remind clinicians of the heterogeneity of our patient populations. In contrast, specialities such as cardiology have made great advances by being able to study more homogeneous populations, subsequently streamlining care. Furthermore, despite a study designed to capture only patients who would require ICU care for at least three days; over one-third of patients had shorter lengths of stay (433 of the 1,200 enrolled). This observation demonstrates the limitation of prediction rules for ICU patients. It also means that it is unclear which patients should have intensive insulin initiated at the time of ICU admission. In addition, this study does have limitations which will raise questions about its widespread applicability. For example, the study was single centred and non-blinded. In addition, although APACHE-II is not an ideal predictor of mortality, the control group's mortality rate was $53 \%$. As such, this work serves as an enticing stimulus for those attempting to better elucidate the aberrant metabolism of critical illness. It should also dampen enthusiasm for those eager to implement a universal protocol for glycemic control in the ICU.

Multivariate regression analysis suggests that blood glucose control, and not the insulin dose, explains most of the beneficial effects on outcome from critical illness. ${ }^{4}$ The mechanisms may include protecting hepatocytic mitchondria, improved polymorphonuclear neutrophil function, and better intracellular bactericidal and opsonic activity. However, putative non-glycemic effects of insulin do exist. These include partial correction of abnormal serum lipids and counteracting the catabolism of critical illness. Improvement in endothelial function, myocardial protection, and decreased inflammation and apoptosis may also contribute. ${ }^{1,3-5}$

In acute critical illness, hepatic glucose production is enhanced by increased gluconeogenesis and glycogenolysis. This occurs despite concomitant increases in insulin production which would normally suppress these pathways. Exercise-stimulated glucose uptake in skeletal muscle decreases dramatically due to patient immobilization. Insulin-stimulated glucose uptake by glucose transporters is also compromised. Total body glucose uptake is increased, but by tissues that are not dependant on insulin, such as the brain and blood cells. Increased counter regulatory hormones, including glucagon, cortisol, growth hormone, catecholamines and cytokines, also play a role. Elevated hepatic glucose production, higher levels of insulin, and impaired peripheral glucose uptake characterize the peripheral insulin resistance of critical-illness. ${ }^{1,3-5}$

If worse outcome in those who received intensive insulin therapy and stayed in ICU less than three days is reproducible, then perhaps short term elevation in glucose is adaptive. Alternatively, hypoglycemia may represent a failure to mount a sufficient stress response via counter-regulatory hormones. Lack of counterregulatory hormones equates to a lack of physiological reserve, and this portends a poor prognosis. ${ }^{6}$ As a result, it would follow that hypoglycemia is an independent risk factor for in-hospital death. Perhaps high dose insulin represents a type of "metabolic stresstest". Notably, there were significantly more hypoglycemic events associated with intensive insulin therapy. 
While both in-hospital and ICU mortality rates were similar when comparing those who experienced hypoglycemia in the conventional $v s$ the intensively treated groups, mortality was higher for both treatment arms when compared to patients who did experience hypoglycemia. Regardless, most ICU physicians are familiar with the work on relative adrenal insufficiency; perhaps another endocrinopathy has been added to our constellation of ICU diseases.

So, what to do? Options include waiting for data from additional trials: whether from a multicentre trial (e.g., the NICE-SUGAR trial) ${ }^{7}$ or one with lower control-group mortality. However, evidence that acute hyperglycemia is more acutely toxic in the critically ill than either healthy or diabetic individuals ${ }^{3-5}$ argues against inaction. Alternatively, the option of treating all medical and surgical patients exists, given the likelihood that more will benefit than by treating none. However, this study illustrates that ICU treatment should be individualized. An interim suggestion is to avoid marked hyperglycemia (serum glucose $>8$ $\mathrm{mmol} \cdot \mathrm{L}^{-1}$ ) for medical ICU patients during the first three days of their admission. If critical illness persists, then subsequent normoglycemia $\left(4.4-6.1 \mathrm{mmol} \cdot \mathrm{L}^{-1}\right)$ becomes justified. ${ }^{6}$

In this study, Van den Berghe et al. provide important new information for the care of medical ICU patients, while at the same time raising additional questions. For those expecting a simple resolution to the question of glycemic control in the critically ill, they will be surprised. For those eager to better characterize the metabolic abnormalities of critical illness, the story just got more fascinating.

Peter G. Brindley MD FRCPC

Damian Paton-Gay MD

University of Alberta, Edmonton, Canada

\section{References}

1 McCowen KC, Malhotra A, Bistrian BR. Endocrine and metabolic dysfunction syndromes in the critically ill. Crit Care Clin 2001; 17: 107-24.

2 Van den Berghe G, Wouters P, Weekers F, et al. Intensive insulin therapy in critically ill patients. N Engl J Med 2001; 345: 1359-67.

3 Vanhorebeek I, Langouche L, Van den Berghe G. Glycemic and nonglycemic effects of insulin: how do they contribute to a better outcome of critical illness? Curr Opin Crit Care 2005; 11: 304-11.

4 Van den Berghe G, Wouters PJ, Bouilllon R, et al. Outcome benefit of intensive insulin therapy in critically ill: insulin dose versus glycemic control. Crit Care Med 2003; 31: 359-66.
5 Langouche L, Van den Berghe G. Glucose metabolism and insulin therapy. Crit Care Clin 2006; 22: 119-29.

6 Malhotra A. Intensive insulin in intensive care (Editorial). N Engl J Med 2006; 354: 516-7.

7 Angus DC, Abraham E. Intensive insulin therapy in critical illness (Editorial). Am J Resp Crit Care Med 2005; 172: 1358-9. 